\title{
GESTIÓN POR PROCESOS Y SU RELACIÓN CON EL PLAN ESTRATÉGICO EN UN CONTEXTO DE MODERNIZACIÓN DE LA GESTIÓN PÚBLICA PERUANA
}

Process management and its Relationship With the STRategic Plan IN THE CONTEXT OF MODERNIZATION OF PUBLIC ADMINISTRATION PERUVIAN

\author{
José Domingo Begazo* \\ d180045@mixmail.com \\ Walter Fernandez-Baca*** \\ wfernandezbaca@pucp.pe
}

[RECEPCIÓN: ABRIL 2016 / CONFORMIDAD: MAYO 2016]

\begin{abstract}
RESUMEN
El presente artículo aborda la importancia y vigencia de la Gestión por Procesos, técnica que brindaría múltiples ventajas en la dirección de las organizaciones, específicamente en la Gestión Pública.

En el Perú, su aplicación constituye un gran reto para encuadrarnos dentro de La Política Nacional de Modernización de la Gestión Pública en el cumplimiento de las exigencias de la Ley 27658.

El artículo pretende fundamentar teóricamente la gestión por procesos como un elemento clave para lograr el éxito organizacional en las instituciones del estado, el método utilizado se basó fundamentalmente en la revisión bibliográfica y el estudio de los métodos teóricos para revelar las relaciones esenciales de la gestión por procesos. Se exponen conceptos actualizados, clasificación, premisas básicas y relaciones de esta categoría. Aporta información de utilidad para el personal directivo que refiere la aplicación de la gestión por procesos, sus generalidades, importancia para la identificación y solución de los problemas, el control continuo sobre los procesos individuales y el propio sistema de procesos, así como su relación con la gestión de la calidad de manera que resalta la incidencia de esta técnica en el éxito de la Gestión Publica.
\end{abstract}

Palabras clave: Gestión por procesos; gestión de calidad; plan estrategico.

\section{ABSTRACT}

This paper addresses the importance and validity of Process Management, a technique that would provide multiple benefits in the management of organizations, specifically in Public Administration. In Peru, its implementation is a major challenge to the power framed within the National Policy Modernization of Public Management in compliance with the requirements of the Law 27658. The document aims to theoretically support the process management as a key element for achieving organizational success in state institution; the method used is mainly based on the literature review

\footnotetext{
* Profesor Principal de la Facultad de Ciencias Administrativas (FCA) de la Universidad Nacional Mayor de San Marcos (UNMSM).

** Profesor de la Pontificia Universidad Católica del Perú y de la Universidad Nacional Mayor de San Marcos (UNMSM).
} 
and study of theoretical methods to reveal the essential relationships of process management. date concepts, classification, basic assumptions and relationships in this category are exposed. Provides useful information for managers referring the application of process management, its generalities importance for identifying and solving problems, continuous control over individual processes and process system itself as well as its relationship with quality management, so that highlights the impact of this technique on the success of Public management

Keywords: Process management; quality management; strategic plan.

\section{INTRODUCCIÓN}

La globalización de los mercados, la apertura económica y el entorno de competencia especialmente en el sector privado ha tenido como estructura básica que los ciudadanos usuarios del servicio público sean más exigentes en el momento de satisfacer necesidades de servicio en la Gestión Pública.

En la administración pública actual la gestión por procesos constituye un reto a cumplir dada las circunstancias actuales del management moderno donde la competitividad rige en el éxito o fracaso de las corporaciones privadas y públicas.

Ante este escenario, el sector público peruano debe de modernizarse con la aplicación del enfoque de Gestión por Procesos tratados en este artículo. Respecto a la importancia de su aplicación el Dr. Zarategli, J.R. (1999) explica: "Los Procesos son secuencias ordenadas y lógicas de actividades de transformación, que parten de unas entradas (informaciones en un sentido amplio, pedidos, datos, especificaciones, medios materiales, etc.) para alcanzar unos resultados programados, que se entregan a quienes los han solicitado, los clientes de cada proceso".

Por lo que una organización publica bien estructurada en base a procesos, facilitaría no solo el control económico, sino además los servicios de calidad a prestarse a los ciudadanos usuarios.

El presente documento constituye una contribución a la solución de esta problemática, pues el mismo tiene como objetivo fundamentar teóricamente la gestión por procesos, como un elemento clave para lograr el éxito en la Gestión pública, donde de manera breve pero didáctica se refiere la aplicación de la gestión por procesos como elemento importante en las organizaciones, útil para el personal directivo y otros profesionales vinculados a procesos específicos en su quehacer diario.

\section{MARCO LEGAL DE APLICACIÓN DE LA GESTIÓN POR PROCESOS}

La Ley № 27658, Ley Marco de Modernización de la Gestión del Estado, declaró al Estado Peruano en proceso de modernización, mediante la Ley № 29158, Ley Orgánica del Poder Ejecutivo, se crea el Sistema de Modernización de la Gestión Pública como un sistema administrativo y precisa que el Poder Ejecutivo tiene la rectoría de los sistemas administrativos, y por Decreto Supremo № 109-2012-PCM, se aprueba la "Estrategia de Modernización de la Gestión Pública 2012-2016". Por ello, el nuevo decreto supremo dispone que el organismo rector del sistema es la Presidencia del Consejo de Ministros, a través de la Secretaría de Gestión Pública. En los niveles subnacionales es de aplicación por los gobiernos regionales, los gobiernos locales y las mancomunidades municipales.

En cuanto al contenido de la norma, ella se inicia con un diagnóstico de la situación general de la gestión pública en el Perú y sus principales deficiencias, plantea los fundamentos de la política con una visión, alcance, objetivos y principios de la misma, sigue con la definición de la estrategia central: una gestión pública orientada a resultados en base a procesos que tenga como uno de sus objetivos claves el servicio al ciudadano (incluyendo componentes, pilares centrales, ejes transversales y desarrollo y sostenibilidad de la política de modernización), avanza en precisar los lineamientos para la modernización y concluye en el rol del ente rector del sistema de modernización de la gestión pública en el Perú.

La inmediatez de organizar las instituciones por procesos, en muchos casos está asociada, entre otros factores, al dominio por parte de los directivos de los elementos básicos de esta técnica, cuestión necesaria a profundizar en la superación actual de directivos del sector público, en sus tres niveles: Nacional, Regional y Local. 


\section{IMPORTANCIA, NECESIDAD Y RELACIÓN DE LA GESTIÓN POR PROCESOS CON LAS NORMAS DE CALIDAD ISO 9000.}

Los procesos son inherentes a toda administración, forman parte de toda empresa ya sea pública o privada; constituyen "lo que se hace y como se hace". Fuerzan a la cooperación y obligan a la creación de una cultura de calidad, más abierta a resultados que al trabajo en base a funciones donde se mantiene privilegios y "zonas de confort” en las instituciones públicas. Fernández Baca, Walter (2015)

"Las normas ISO 9000 consiste en instaurar en una empresa, un conjunto de requerimientos para inyectar a sus procesos de transformación, las características necesarias para elaborar un producto o servicio que satisfaga las especificaciones previamente acordadas con el usuario de manera consistente". Representan el marco hacia la mejora del desempeño del sistema de gestión de calidad de una organización. Se presentan ocho principios fundamentales, uno de ellos se refiere al Enfoque por Procesos, el cual plantea que un "resultado deseado se alcanza más eficientemente cuando las actividades y los recursos relacionados se gestionan como un proceso."

\section{PRINCIPIOS DE LA CALIDAD}

\section{PRINCIPIOS}

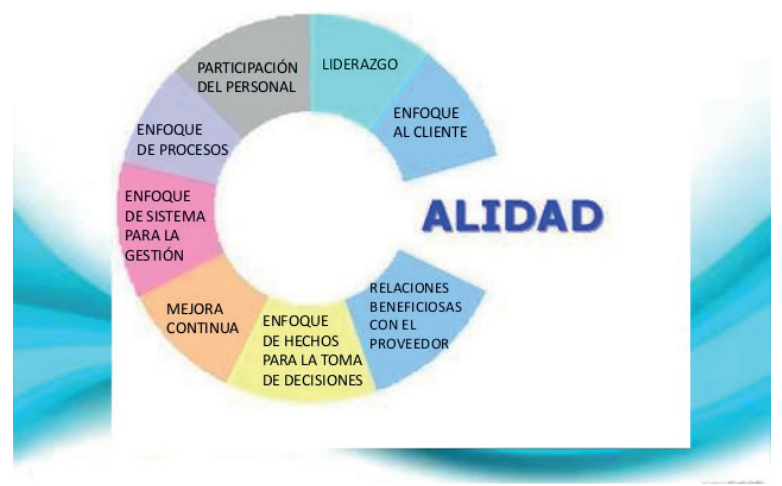

Figura 1 - Elaboración propia según Norma UNE-EN ISO 9000:2000

Estos principios en el cual encontramos el enfoque de procesos se fundamentan en la familia de normas internacionales cuya finalidad es la de conducir y operar una organización de forma exitosa a través de una gestión sistemática y transparente.

De acuerdo a la norma arriba indicada, el enfoque basado en procesos es el logro de todos los objetivos planteados por la organización se consigue considerando todos los recursos y las actividades de la empresa como procesos, gestionando la calidad a través de los procesos identificados.

Hoy en día, las técnicas más actualizadas en Gestión Empresarial reservan un lugar especial a los conceptos de actividad y de procesos. El éxito de toda organización depende, cada vez más, de que sus procesos empresariales respondan a su estrategia, misión y objetivos.

Detrás del cumplimiento de un objetivo, se encuentra la realización de un conjunto de actividades que, a su vez, forman parte de un proceso. Es por ello que el principal punto de análisis lo constituye, precisamente, la gestión de la empresa basada en los recursos y los procesos que la integran. Un resultado deseado se alcanza con más eficiencia cuando sus actividades y recursos relacionados, son manejados como procesos.

El enfoque orientado hacia los procesos, permite una rápida y sencilla identificación de los problemas. Así como la rápida solución de los mismos, la que se puede realizar sin la necesidad de mejorar el resto de los procesos.

De manera que, la modificación o cambio de un proceso, no lleva aparejada la modificación o cambio del resto de los procesos, pero si sobre su resultado final, por lo que todos los procesos, han de cumplir con las expectativas y necesidades del resto de los procesos por estar integrados. La responsabilidad de la mejora de un proceso, corresponde a sus integrantes directos, pero siempre con la ayuda de toda la organización.

Por su parte, la ISO 9000: 2015 ofrece una visión generalizada y aplicable a cualquier actividad, pues enuncia como "un proceso a toda aquella actividad que utiliza recursos y que se gestiona con el fin de permitir que los elementos de entrada se transformen en resultados"/. El sistema por procesos, es más fácil de implementar, y más económico de mantener en correcto funcionamiento. Tiene la ventaja, de que aunque un proceso afecte al resto, resulta más sencillo para cambiar este o partes de la cadena, sin que el resto se vea afectado de forma negativa por la transformación.

En la actualidad varios municipios de Lima y diversas entidades públicas ya cuentan con la certificación de calidad correspondiente, sobre la base de los procesos internos normados a nivel internacional; ya que constituye una de las premi- 
sas básicas para lograr la "Gestión de la Calidad Total», que busca la satisfacción de los ciudadanos usuarios de los servicios públicos.

\section{GESTIÓN DE LOS PROCESOS Y PLAN ESTRATÉGICO}

Según directivas del Centro Nacional de Planeamiento Estratégico Directivas 001-2014- CEPLAN "la articulación de los Planes Regionales y Locales al Plan Bicentenario es de cumplimiento obligatorio". Por ello determinamos que el enfoque basado en procesos exige una orientación al cumplimiento de los Planes Estratégicos y la mejora continua según CEPLAN. Cuando en las organizaciones se trabaja de manera operativa en función del cumplimiento de metas, sin el estudio de manera detallada de cada uno de los elementos estructurales y sus posibles deficiencias, puede dar como resultado la aparición de procesos fuera de control, al no considerar las limitaciones inherentes al proceso, y por tanto genera ineficiencia e ineficacia y la correspondiente ausencia de calidad.

Con el enfoque basado en procesos resulta obvio el cumplimiento de las metas y objetivos del Plan Estratégico en sus diversas fases como dictamina la figura anterior. Observamos que los Planes de Desarrollo concertado y los llamados
POI (Plan Operativo Institucional), de los Gobiernos regionales y locales deben de presumir de la correcta evaluación de los diferentes procesos de una organización y que son factibles de planificar, controlar y mejorar; y por tanto evaluarse la capacidad real y objetiva, para entregar una salida en función de sus entradas y de la forma en que se gestiona.

El Plan Estratégico es de suma importancia, se observa que es el Pilar Central № 1, en las Política Nacional de Modernización de la gestión Pública, por ello resulta de necesidad incorporar la gestión de Procesos desde la Planeación Estratégica. Por medio de los procesos se lograra la alineación de los mismos con la estrategia, misión y objetivos, como un gran sistema interrelacionado destinados a incrementar la satisfacción de nuestros ciudadanos usuarios de nuestro servicio, el incremento del llamado valor público y la capacidad de respuesta del estado en su labor de satisfacer necesidades del ciudadano.

En la actualidad el enfoque y gestión de los procesos de acuerdo al criterio de varios autores es la base para entender la organización como un sistema, superar las contradicciones interdepartamentales y eliminar los problemas de diseño estructural como desbalances entre responsa-

Fases del proceso de planeamiento estratégico

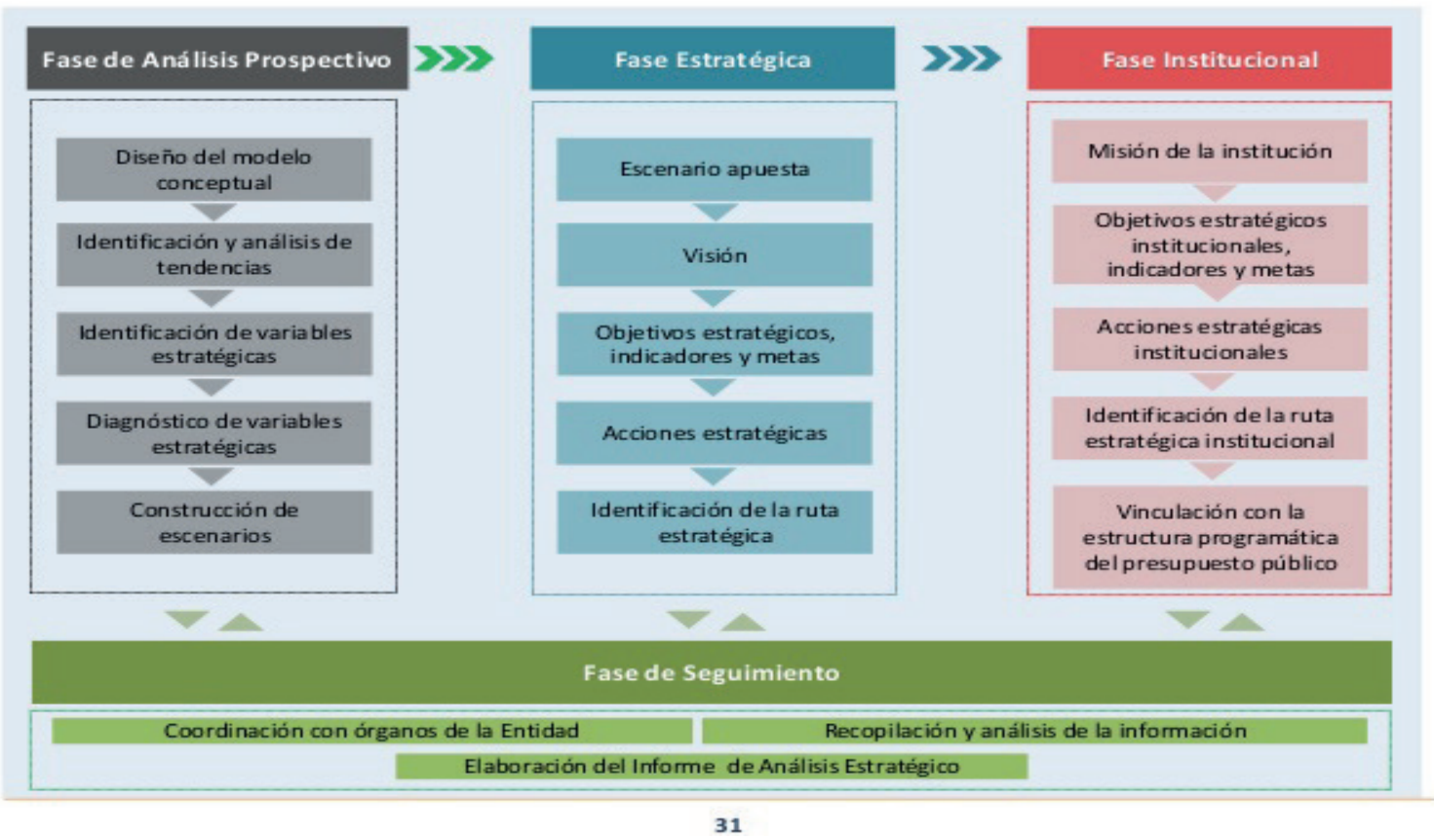

Figura 2. Elaboración: Ceplan 


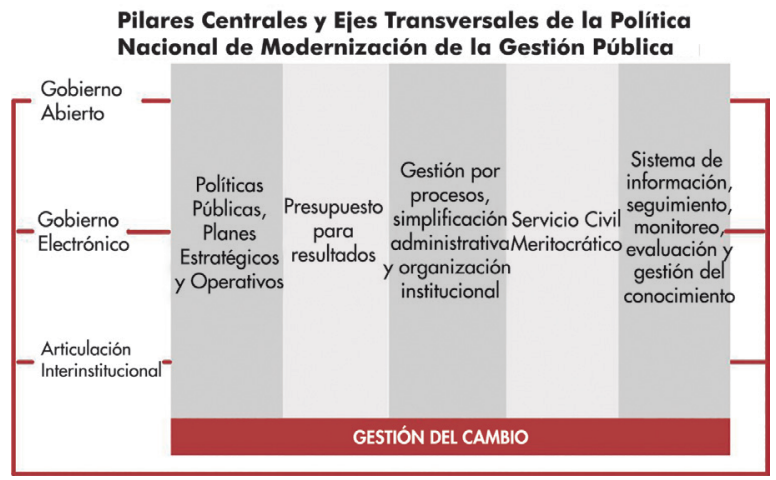

Figura 3. Elaboración: Ceplan

bilidad y autoridad, confusiones entre las líneas ejecutivas y funcionales, insuficiente manejo de estructuras paralelas, desproporciones entre áreas de dirección, exceso, defecto o redundancia de funciones, o sus agrupaciones erróneas al aplicar uno u otro criterio de departamentalización.

\section{BALANCED SCORECARD Y GESTIÓN POR PROCESOS}

El estudio del Balanced Scorecard, también llamado Cuadro de Mando Integral es de suma importancia, su implementación creemos se hace impostergable en la moderna Gestión Publica.

Según Robert Kaplan (2012) uno de sus creadores: "Es una herramienta que permite enlazar estrategias y objetivos clave con desempeño y resultados a través de 4 áreas críticas en cualquier empresa: Desempeño Financiero, conocimiento del cliente, Procesos Internos y aprendizaje y crecimiento".

La relación existente entre el Cuadro de Mando Integral (CMI) y la Gestión por Procesos está dada desde su coincidencia en el tiempo y propósitos, hasta la existencia de la dimensión "Procesos" en el CMI.

El análisis del valor añadido en los procesos se realiza mediante su descomposición en pasos o etapas y posteriormente, se debe realizar un estudio de cada una y se clasifican las actividades que aportan o no valor añadido para considerar su posible eliminación o mejoramiento.

Mientras mayor sea la capacidad del proceso para cumplir con los resultados deseados, más eficaz será. Los recursos utilizados son parte del precio para cumplir con los objetivos y metas deseadas. Cuanto menor sea la cantidad de recursos necesarios para lograr los objetivos y resultados, más eficiente será el proceso.

Es necesario destacar la necesidad de la gestión por Procesos desde un concepto holístico, la mejora de un proceso eleva la calidad y la satisfacción de nuestros ciudadanos usuarios del servicio, también mejora el desarrollo de habilidades blandas en los colaboradores o servidores públicos, la incorporación del Benchmarking o

\section{Las 4 perspectivas del BSC}

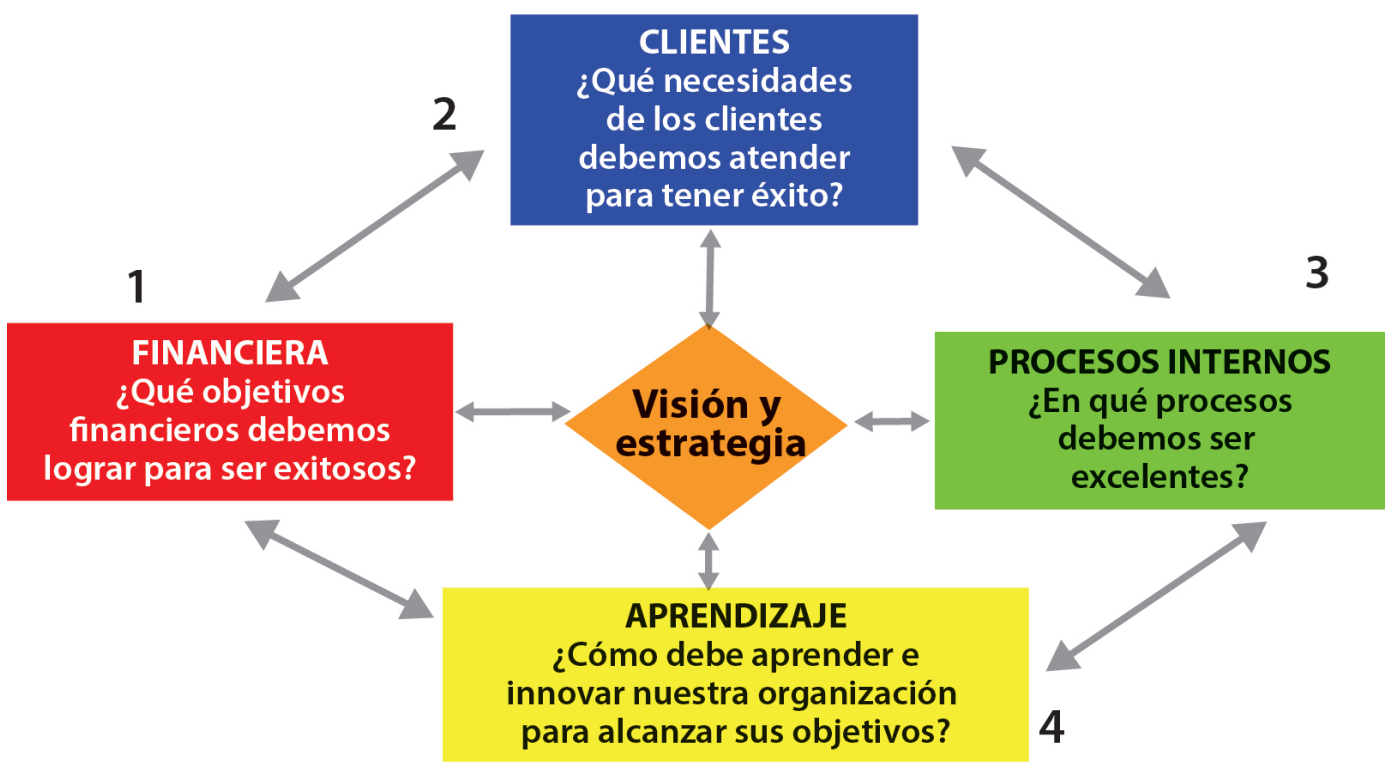

Figura 4. Elaboración: Kaplan \& Norton 
el diseño de mejores prácticas de excelencia es otra variable a tener en cuenta con esta práctica.

\section{CONCLUSIONES}

1 Para responder a las necesidades de un entorno cambiante, la Administración Publica tiene que trabajar en base a la Gestión por Procesos. Ya no se piensa que los procesos puedan diseñarse con una estructura ideal, que vaya a permanecer inmutable con los pasos de los años. Por el contrario, los procesos están permanentemente sometidos a revisiones en el cual los colaboradores o servidores públicos deben estar comprometidos con ella.

2 Cada día se cobra mayor conciencia por parte de todos los componentes del sistema de que el logro de eficiencia, la eficacia y el cumplimiento de la misión debe de mejorar en forma continua en las instituciones públicas.

Para que en una organización del estado se pueda implantar correctamente la gestión por procesos, la organización deberá invertir tiempo y esfuerzo, participación y formación. Debe tenerse en cuenta que toda actividad o secuencia de actividades que se llevan a cabo en las diferentes unidades constituye un proceso y como tal, hay que gestionarlo. La reciente Ley SERVIR que tiene como uno de sus objetivos la Meritocracia, será un aliado muy importante en la Gestión del Talento Humano en las organizaciones públicas.

El impulso hacia la transformación y el desarrollo organizacional en la práctica cotidiana, avala reconocer a los procesos como técnica ligada a la gestión de la calidad.

En la gestión por procesos el significado más acertado para el concepto calidad es: lo que el cliente o ciudadano usuario espera recibir por lo que está dispuesto a pagar en función del valor percibido. Desde este punto de vista la calidad equivale a «orien- tación de la empresa hacia el cliente»; por lo que la gestión por procesos se vincula a la gestión de la calidad para lograr la calidad Total. Enfoque propuesto por la norma de calidad ISO 9001.

6 En el sector público la aplicación del enfoque de procesos es viable y conduce a elevar la calidad de los servicios, su eficacia y eficiencia. Esta técnica da la posibilidad para cumplir con las exigencias de los diversos Lineamientos en materia de Política nacional de Modernización de la Gestión Publica y otras normas dadas con la finalidad de mejorar el servicio público.

\section{REFERENCIAS BIBLIOGRÁFICAS}

Zarategli, J.R. (1999) La gestión de procesos; su papel e importancia en la empresa. Economía Industrial España Arellano, Rolando "Marketing para Vivir Mejor" Editorial Planeta 2013 Lima

Fernández Baca (2015). Módulo de estudios Diplomado en Administración de Calidad en la Gestión Pública \& Escuela Nacional de Gestión Pública ENEG 2015 Lima.

Centro Nacional de Planeamiento EstratégicoCEPLAN PERU Documentos diversos - Plan Bicentenario actualizado - 2014 - Características.

Sánchez Vásquez, Vicente - Abogado PUCP, Blog personal - Temas de gestión Publica 2016

Negrin E. Metodología para el perfeccionamiento de los procesos en empresas hoteleras. CICAG [Internet]. 2003 [citado 12 enero 2013]; http://www.publicaciones.urbe.edu/ index.php/cicag/article/view/547/1346

Medina, Nogueira y Hernández (2012) Relevancia de la Gestion Por Procesos en la Planificación Estratégica y Mejora continua - Revista UMCC

Marrero A.(2010) La gestión por Procesos en Organizaciones de Salud - Revista Universidad de la Juventud- Cuba. 